\title{
Exercício físico na prevenção de quedas do idoso da comunidade: revisão baseada na evidência
}

\author{
Physical exercise for preventing falls in elder people living in the community \\ Ejercicio físico en la prevención de caídas del anciano de la comunidad: revisión basada \\ en la evidencia \\ Joana Rita Bento, Nadina Duarte Sousa
}

\section{Resumo}

Introdução: A prevenção de quedas do idoso é um urgente desafio de saúde pública. O exercício físico tem-se comprovado uma intervenção preventiva eficaz. Objetivo: Rever a evidência científica mais recente, relativa à recomendação do exercício físico na prevenção de quedas do idoso da comunidade. Identificar modalidade física e regimes de frequência com maior benefício. Métodos: Efetuou-se uma pesquisa bibliográfica, utilizando os termos MeSH "aged", "accidental falls/prevention and control" e "exercise", nas bases de dados PubMed, Cochrane Library, National Guideline Clearinghouse, Canadian Medical Association, Evidence based Medicine e NICE Evidence Search, nos últimos 5 anos. Foram incluídos artigos que avaliassem o exercício como intervenção isolada na prevenção de quedas do indivíduo da comunidade, $c 0 m \geq 60$ anos. Resultado avaliado: incidência de quedas. Resultados: De 637 potenciais artigos, 4 cumpriam critérios de inclusão: 2 ensaios clínicos aleatorizados controlados (ECACs), 1 estudo coorte e 1 meta-análise (MA). Globalmente, os resultados demonstram um efeito protetor do exercício físico. A MA obteve uma razão de taxas de incidência $(R T I)$ de $0,79(p<0,001)$, com o treino de equilíbrio $(R T I=0,85, p=0,04)$ e maior dose semanal $(R T I=0,77, p=0,03)$ a demonstrarem-se mais efetivos. Contrariamente, um ECAC demonstrou superioridade na marcha. $O$ estudo coorte revelou maior benefício em participações $\geq 3$ anos $(R T I=0,90 ; p=0,03)$. Conclusão: $O$ exercício físico é efetivo na prevenção de quedas do idoso da comunidade. Treinos de equilíbrio e práticas semanais $\geq 3$ horas demonstraram melhores resultados (SORT A). A sua manutenção deve ser incentivada a longo prazo (SORT B).

\section{Abstract}

Introduction: The prevention of falls among elder people is an urgent public health challenge. Physical exercise has proven an effective preventive intervention. Objective: Review the latest scientific evidence, concerning the recommendation of physical exercise in the prevention of falls of the elderly of the community. Identify the optimal type and frequency of exercise with better benefits. Methods: A bibliographic search was carried out using the terms MeSH "aged" , "accidental falls/prevention and control" and "exercise", in PubMed, Cochrane Library, National Guideline Clearinghouse, Canadian Medical Association, Evidence based Medicine e NICE Evidence Search databases, in the last five years. We included articles evaluating the exercise as an isolated intervention in the prevention of falls of the individual of the community, with $\geq 60$ years. Rated result: incidence of falls. Results: Of 637 potential articles, 4 fulfilled inclusion criteria: 2 randomized controlled trials (RCT), 1 cohort study and 1 meta- analysis (MA). Overall, the results demonstrate a protective effect of exercise. The MA obtained a ratio of incidence rates (IR) was $0.79(p<0.001)$, with the training of balance $(I R=0.85, p=0.04)$ and greater weekly dose $(I R=0.77, p=0.03)$ has demonstrated more effective. Contrarily, one RCT suggested that hike can be more effective. The cohort study revealed greater benefit in $\geq 3$ years participations ( $\mathrm{IR}=0.90 ; p=0.03$ ). Conclusion: Physical exercise is effective in preventing falls in the community elder. Balancing training and weekly $\geq 3$ hours practices showed better results (SORT A). Its maintenance must be encouraged in the long term (SORT B).

\section{Palavras-chave:}

Acidentes por Quedas Acidentes por Quedas/ prevenção \& controle Exercício Físico Idoso

Keywords:

Falls

Falls/prevention \& control Physical Exercise Aged
Como citar: Bento JR, Sousa ND. Exercício físico na prevenção de quedas do idoso da comunidade: revisão baseada na evidência. Rev Bras Med Fam Comunidade. 2017;12(39):1-11. http://dx.doi.org/10.5712/rbmfc12(39)1658

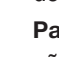

não se aplica.

Conflito de interesses:

declaram não haver.

Procedência e revisão por pares: revisado por pares.

Recebido em: 19/11/2017.

Aprovado em: 02/01/2018. 


\section{Resumen}

Introducción: La prevención de caídas del anciano es un urgente desafío de salud pública. El ejercicio físico se ha comprobado una intervención preventiva eficaz. Objetivo: Revisar la evidencia científica más reciente, relativa a la recomendación del ejercicio físico en la prevención de caídas del anciano de la comunidad. Identificar modalidad física y regímenes de frecuencia con mayor beneficio. Métodos: Se realizó una investigación bibliográfica, utilizando los términos MeSH "aged", "accidental falls/prevention and control" y "exercise", en las bases de datos PubMed, Cochrane Library, National Guideline Clearinghouse, Canadian Medical Association, Evidence based Medicine y NICE Evidence Search, en los últimos 5 años. Se incluyeron artículos que evaluasen el ejercicio como intervención aislada en la prevención de caídas del individuo de la comunidad, con $\geq 60$ años. Resultado evaluado: incidencia de caídas. Resultados: De 637 potenciales artículos, 4 cumplían criterios de inclusión: 2 ensayos clínicos aleatorizados controlados (ECACs), 1 estudio cohorte y 1 meta-análisis (MA). Globalmente, los resultados demuestran un efecto protector del ejercicio físico. La MA obtuvo una proporción de tasas de incidencia (RTI) de $0,79(p<0,001)$, con el entrenamiento de equilibrio $(R T l=0,85, p=0,04)$ y la mayor dosis semanal $(R T I=0,77, p=0,03)$ se demostraron más efectivos. Contrariamente, un ECAC demostró superioridad en la marcha. El estudio cohorte reveló un mayor beneficio en participaciones $\geq 3$ años $(\mathrm{RTI}=0,90, p=0,03)$. Conclusión: El ejercicio físico es efectivo en la prevención de caídas del anciano de la comunidad. Los entrenamientos de equilibrio y las prácticas semanales $\geq 3$ horas demostraron mejores resultados (SORT A). Su mantenimiento debe ser incentivado a largo plazo (SORT B).
Palabras clave:

Accidentes por Caídas

Accidentes por Caídas/ prevención \& control Ejercicio Físico Anciano

\section{Introdução}

Registrando-se uma tendência crescente na proporção da população mundial com 65 ou mais anos, ${ }^{1}$ o envelhecimento populacional representa um dos fenômenos demográficos mais preocupantes das sociedades modernas. ${ }^{2}$

Na perspectiva biológica, o envelhecimento está associado à acumulação de danos moleculares e celulares, conduzindo a uma perda gradual nas reservas fisiológicas, a um aumento do risco de contrair doenças e a um declínio geral na capacidade intrínseca do indivíduo; em última instância, resulta no falecimento. Não obstante, essas mudanças não são lineares ou consistentes, e estão apenas vagamente associadas à idade de uma pessoa em anos. ${ }^{3}$ Em Portugal, consideram-se pessoas idosas os homens e as mulheres com idade igual ou superior a 65 anos. ${ }^{1}$ Já no Brasil, esta definição tem início a partir dos 60 anos. $^{4}$

São diversas as síndromes geriátricas, nas quais se destacam as quedas como problema clínico de elevado impacto do idoso. ${ }^{5}$ Esta pertinência é resultado da combinação de uma elevada incidência, com uma elevada suscetibilidade à lesão, dada a prevalência aumentada de doenças crônicas e alterações fisiológicas relacionadas com a idade; ainda, a recuperação da queda em indivíduos idosos é tipicamente retardada, conduzindo a um consequente declínio funcional. ${ }^{6}$

As quedas são definidas pela American Geriatrics Society (AGS) e pela British Geriatrics Society (BGS) como um contato não intencional com a superfície de apoio, resultante da mudança de posição do indivíduo para um nível inferior à sua posição inicial, sem que tenha havido fator intrínseco determinante ou acidente inevitável e sem perda de consciência. ${ }^{7}$ Estima-se que este fenômeno atinja $30 \%$ dos adultos com mais de 65 anos e $40 \%$ após os 80 anos, ${ }^{8}$ sendo responsável por $87 \%$ das fraturas, $50 \%$ dos internamentos em idosos, e por um elevado consumo de recursos em saúde. ${ }^{3}$

Evidência na literatura aponta para a existência de 400 fatores de risco para quedas, que contemplam as dimensões biológica, comportamental, ambiental e socioeconômica e que interagem entre si, aumentado a probabilidade de ocorrência com o aumento do número de variáveis associadas. ${ }^{9}$

A prevenção de quedas e suas consequências é um urgente desafio de saúde pública internacional. ${ }^{10}$ Várias intervenções têm sido descritas: abordagem multifatorial dos fatores de risco identificados, programas 
de exercício físico, revisão terapêutica, correção de deficiências visuais, tratamento da hipotensão postural, abordagem de alterações do ritmo e de frequência cardíaca, tratamento de afecções do pé, modificação de fatores de risco ambientais do domicílio e a educação do indivíduo. ${ }^{7}$

Atualmente, diversos estudos e revisões sistemáticas evidenciam o papel dos programas interventivos na prevenção de quedas. Uma recente revisão sistemática de Cochrane concluiu que as intervenções de exercício físico compostas por diferentes tipos de exercícios, em grupo, ou individuais, praticadas no domicílio, reduzem o risco e incidência de quedas (Razão de taxas de incidência (RTI) de 0,71 e 0,68, respectivamente); especificamente, para a modalidade de Tai Chi, o seu benefício parece ser superior nos indivíduos sem elevado risco de queda. ${ }^{11}$ Já em 2010, a AGS e a BGS recomendavam, com uma força de recomendação $A$, oferecer ao idoso em risco de queda um programa de exercício físico que incorporasse treino de equilíbrio, marcha e de fortalecimento; adicionalmente, treinos de flexibilidade e resistência poderiam ser oferecidos, ainda que não de forma isolada. ${ }^{7}$

Este estudo tem como objetivo rever a evidência científica mais recente, relativa à recomendação do exercício físico na prevenção de quedas do idoso da comunidade, procurando identificar a modalidade física, duração, intensidade e regimes de frequência com maior benefício.

\section{Métodos}

Efetuou-se uma pesquisa bibliográfica, em 7 de outubro de 2017, utilizando os termos MeSH "aged", "accidental falls/prevention and control" e "exercise". Pesquisaram-se normas de orientação clínica, estudos coorte, ensaios clínicos aleatorizados controlados (ECACs), meta-análises (MA) e revisões sistemáticas publicadas nos últimos 5 anos, nas línguas portuguesa e inglesa, nas bases de dados PubMed, Cochrane Library, National Guideline Clearinghouse, Canadian Medical Association, Evidence based Medicine e NICE Evidence Search.

Foram incluídos artigos que avaliassem ou recomendassem o exercício físico como intervenção para a prevenção de quedas do indivíduo da comunidade, com idade igual ou superior a 60 anos. $O$ resultado avaliado foi a incidência de quedas. Constituíram critérios de exclusão os artigos em que a incidência de quedas não correspondesse ao outcome primário, que não avaliassem o exercício físico como intervenção isolada, populações residentes em lar, hospitalizados ou em programas de reabilitação pós-internamento, idosos que padecem de morbilidades específicas: demências e deterioração cognitiva, fragilidade, fratura do colo do fêmur, doença neuromuscular, musculoesquelética ou cardiovascular graves, e artigos com metodologia de menor qualidade. A presença prévia de queda não constituiu um critério de exclusão.

Para avaliação dos níveis de evidência e atribuição de forças de recomendação, foi utilizada a escala Strenght of Recommendation Taxonomy (SORT) da American Academy of Family Physicians.

\section{Resultados}

Da pesquisa bibliográfica resultou um total de 637 potenciais artigos, dos quais apenas 4 cumpriam os critérios de inclusão: 2 ECACs, 1 estudo coorte e uma MA (Figura 1). A descrição dos artigos encontra-se resumida nos Quadros 1, 2 e 3, respectivamente. 


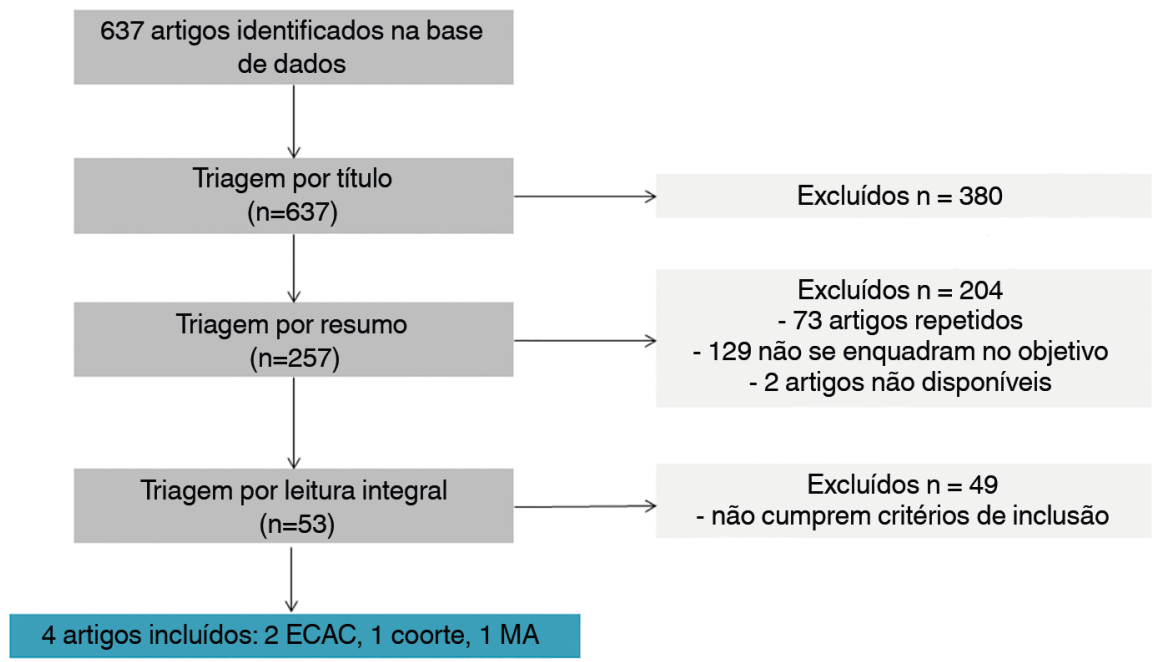

Figura 1. Fluxograma de seleção dos estudos incluídos. ECAC: ensaio clínico aleatorizado controlado; MA: meta-análise.

Quadro 1. Ensaios clínicos controlados aleatorizados.

\begin{tabular}{|c|c|c|c|c|}
\hline Referência & Metodologia & Resultados & Conclusões & NE \\
\hline $\begin{array}{l}\text { Gawler et al. }{ }^{12} \\
(2016)\end{array}$ & $\begin{array}{l}\text { Estudo prospectivo, } \\
\text { Inglaterra } \\
1256 \text { indivíduos da comunidade, com } \\
\text { idade [65-94] anos; } 84 \%<80 \text { anos } \\
62 \% \text { sexo feminino } \\
\text { - Programa de exercício FaME } \\
\text { ( } n=387 \text { ) } \\
\text { - Programa de exercício OEP ( } n=411 \text { ) } \\
\text { - CH ( } n=458 \text { ) } \\
\text { Resultados avaliados: incidência de } \\
\text { quedas e razão de taxas, aos } 12 \text { e } 24 \\
\text { meses }\end{array}$ & $\begin{array}{l}\text { Taxa de incidência (quedas/pessoa-ano): } \\
12 \text { meses } \\
\text { >FaME: } 0,53 \\
\text { >OEP: } 0,53 \\
\text { >CH: } 0,69 \\
24 \text { meses } \\
\text { >FaME: } 0,42 \\
\text { >OEP: } 0,53 \\
\text { >CH: } 0,46 \\
\text { Comparação da taxa de incidência em } \\
\text { relação ao grupo de CH: } \\
12 \text { meses } \\
\text { >FaME: RTI=0,74, 95\% IC 0,55-0,99, } \\
\text { p=0,04 } \\
\text { >OEP: RTI=0,76, 95\% IC 0,53-1,09, p=0,14 } \\
24 \text { meses } \\
\text { >FaME: } R T I=0,94,95 \% \text { IC 0,62-1,41, } \\
\text { p=0,76 } \\
\text { >OEP: } \text { RTI=1,04, 95\% IC 0,69-1,55, p=0,86 }\end{array}$ & $\begin{array}{l}\text { A intervenção FaME parece } \\
\text { exercer um efeito protetor de } \\
\text { quedas, além do período de } \\
\text { intervenção de } 24 \text { semanas. } \\
\text { Este efeito é perdido ao segundo } \\
\text { ano de seguimento, pelo que a } \\
\text { manutenção de atividade física } \\
\text { moderada a intensa deve ser } \\
\text { incentivada na prevenção de } \\
\text { quedas a longo prazo. }\end{array}$ & 2 \\
\hline $\begin{array}{l}\text { Okubo et al. }{ }^{13} \text {, } \\
(2016)\end{array}$ & $\begin{array}{l}\text { Estudo prospectivo } \\
\text { Japão } \\
\text { Indivíduos [65-79] anos e baixo risco } \\
\text { de queda }(\mathrm{n}=90) \\
\text { - Grupo marcha ( } \mathrm{n}=50) \text { : } \\
\text { - Grupo treino de equilíbrio ( } \mathrm{n}=40) \\
\text { Resultados primários: } \\
\text { - № quedas } \\
\text { - № tropeços } \\
\text { Follow-up: } 3 \text { meses de exercício su- } \\
\text { pervisionado, seguido de } 13 \text { meses } \\
\text { de exercício não supervisionado }\end{array}$ & $\begin{array}{l}\text { Comparação da taxa de incidência de } \\
\text { quedas do grupo de marcha com o grupo } \\
\text { de treino de equilíbrio, segundo exposição: } \\
\text { >RTI=0,38 quedas/pessoa ativa/dia, } 95 \% \\
\text { IC 0,19-0,77, } p=0,008 \\
\text { >RTI=0,47 quedas/passos/por pessoa, } \\
95 \% \text { IC } 0,26-0,85 ; p=0,012 \\
\text { Comparação da taxa de incidência de } \\
\text { tropeços do grupo de marcha com o grupo } \\
\text { de treino de equilíbrio, segundo exposição: } \\
\text { >RTI=1,66 pessoa-ano, } 95 \% \text { IC 1,34-2,05, } \\
\text { p<0,0001 } \\
\text { >RTI=1,50/pessoa ativa/dia, } 95 \% \\
\text { IC 1, } 12-2,00, p=0,008\end{array}$ & $\begin{array}{l}\text { A marcha pode ser mais efetiva } \\
\text { na prevenção de quedas que o } \\
\text { treino de equilíbrio, nos idosos } \\
\text { da comunidade. Contudo, pode } \\
\text { induzir mais tropeços, pelo que } \\
\text { não deve ser recomendada } \\
\text { no idoso com elevado risco de } \\
\text { queda. }\end{array}$ & 2 \\
\hline
\end{tabular}

FaME: Falls management exercise; OEP: Otago exercise program; $\mathrm{CH}$ : Cuidados habituais; RTI: Razão de taxas de incidência. 
Quadro 2. Estudo coorte.

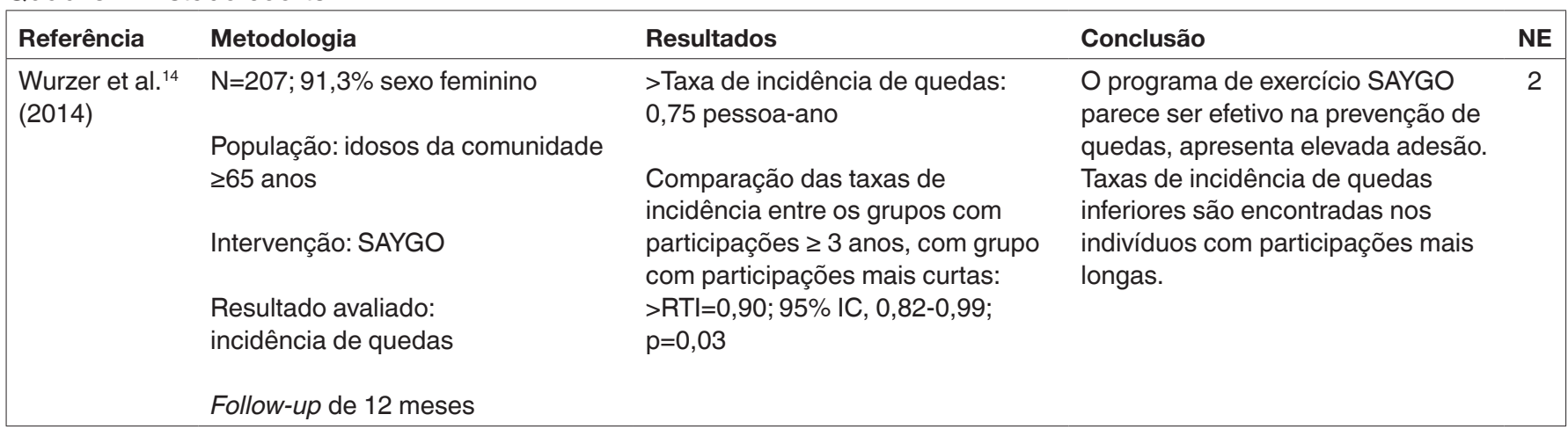

SAYGO: Steady as You Go.

Quadro 3. Meta-análise.

\begin{tabular}{|c|c|c|c|c|}
\hline Referência & Metodologia & Resultados & Conclusão & NE \\
\hline $\begin{array}{l}\text { Sherrington et al. }{ }^{15} \\
(2017)\end{array}$ & $\begin{array}{l}\text { N=61 (ECAC), } 69 \text { comparações } \\
\text { População: indivíduos idosos } \geq 65 \\
\text { anos ( } \mathrm{n}=15 \text { 773) } \\
\text { Intervenção: exercício físico como } \\
\text { intervenção isolada na prevenção } \\
\text { de quedas vs. grupo controle } \\
\text { Resultados avaliados: taxa de } \\
\text { incidência de quedas }\end{array}$ & $\begin{array}{l}\text { RTI=0,79, 95\% IC 0,73-0,85, } p<0,001, \\
\mathrm{I}^{2} 47 \% \\
\text { ECACs com treino de equilíbrio de } \\
\text { elevada dificuldade: } \\
\mathrm{RTI}=0,85,95 \% \text { IC } 0,73-0,995, \mathrm{p}=0,04 \\
28 \% \text { da heterogeneidade explicada } \\
\text { ECACs com } \geq 3 \text { h de exercício semanais: } \\
\mathrm{RTI}=0,77,95 \% \text { IC } 0,65-0,91, p=0,03 \\
61 \% \text { da heterogeneidade explicada }\end{array}$ & $\begin{array}{l}\text { O exercício físico, como } \\
\text { intervenção única, previne } \\
\text { as quedas no idoso. } \\
\text { Programas de exercício } \\
\text { que contemplem treinos } \\
\text { de equilíbrio e uma dose } \\
\text { maior apresentam melhores } \\
\text { resultados. }\end{array}$ & 1 \\
\hline
\end{tabular}

ECAC: Ensaio clínico aleatorizado controlado; RTI: Razão de taxas de incidência.

\section{Ensaios clínicos aleatorizados controlados}

Gawler et al. ${ }^{12}$ realizaram um ensaio clínico randomizado por cluster, multicêntrico, intitulado "ProAct65+", que avaliava dois programas de exercício - "Falls management exercise" (FaME) e "Otago exercise program" (OEP), na prevenção de quedas do indivíduo idoso, inativo, de baixo risco, recomendados no Reino Unido pelo Department of Health Preventing Package.

Ambos são desenhados para aplicação na comunidade, em indivíduos com idade igual ou superior a 65 anos: o primeiro, um exercício de grupo realizado sob orientação de um instrutor, assenta em exercícios de fitness e nos princípios de programas orientados para o idoso (ex: aquecimento, mobilidade alongamentos, força e equilíbrio, resistência e arrefecimento). O OEP é realizado no domicílio, inclui um pequeno aquecimento e exercícios de força e equilíbrio adequados à idade, tendo-se comprovado custoefetivo nos grupos etários acima dos 80 anos.

Mil duzentos e cinquenta e seis pacientes entre os 65 e 94 anos foram recrutados e randomizados no grupo FaME ( $n=387)$, OEP ( $n=411)$ e num grupo de cuidados habituais $(C H)(n=458)$, em que era permitido que praticassem outros exercícios, tal como fariam fora do contexto do estudo. Com uma duração de 24 semanas, a intervenção FaME consistia uma sessão semanal de 1 hora, com um instrutor, suplementada com 30 minutos de exercícios no domicílio; na OEP eram praticados 30 minutos de exercício no domicílio, 3 vezes por semana. Aos idosos, era solicitado o preenchimento de um diário de quedas, ao longo da intervenção; nos 2 anos seguintes, eram contatados trimestralmente, para que reportassem as quedas decorridas nos 3 meses prévios. 
Aos 12 meses de follow-up após suspensão da intervenção, verificou-se uma redução de $26 \%$ das quedas no grupo FaME relativamente ao grupo de cuidados habituais ( $R T I=0,74$, IC 95\% 0,59-0,99; $p=0,04$ ) e uma redução não significativa de $24 \%$ no grupo OEP (RTI=0,76, IC 95\% 0,53-1,05; $p=0,14)$. Aos 2 anos pós-intervenção, o efeito verificado foi perdido (FaME: RTI=0,94, IC 95\% 0,62-1,41; p=0,76; OEP: RTI= 0,76, IC 95\% 0,53-1,05; $p=0,14)$.

Uma análise post-hoc efetuada para investigação desta perda de efeito no grupo FaME revelou, quando da comparação de indivíduos deste grupo que mantinham a prática de 150 minutos semanais de exercício físico moderado a vigoroso aos 2 anos após intervenção, com idosos que já não mantinham a prática de exercício físico, a persistência de resultados estatisticamente significativos (RTI=0,49, IC 95\% 0,30-0,79; $p=0,004)$.

Com este trabalho, os autores demonstraram que o programa de exercício FaME reduz a incidência de quedas no individuo idoso da comunidade, sendo que este efeito é perdido aos 2 anos pós-intervenção, se níveis de atividade física moderados a intensos não forem mantidos. Já o programa OEP parece ser menos efetivo nesta população de baixo risco de queda.

Uma revisão de Sherrington et al., ${ }^{10}$ que incluía 44 ECACs, demonstrou um risco aumentado de quedas com a prática de exercícios de marcha, contudo, esta população incluía indivíduos com elevado risco de queda, pelo que Okubo et al. $^{13}$ conduziram um estudo que testou a hipótese da utilização de exercício de caminhada na prevenção de quedas do indivíduo de baixo risco, no ambulatório. Para esse efeito, selecionaram um total de 90 indivíduos com idades compreendidas entre os 65 e 79 anos, que aleatorizaram num programa de caminhada $(n=50)$ e num programa de treino de equilíbrio $(n=40)$ inicialmente supervisionados por um período de 3 meses, seguido de um período de 13 meses não vigiado, no Japão.

Os programas de intervenção consistiam em sessões semanais, com 2 horas de duração, ao longo de 12 semanas. O exercício primário no grupo de caminhada era composto por marcha rápida numa estrada pedonal, com aumento gradual da distância e velocidade; era ainda recomendada a prática de caminhada no domicílio, 30-50 min, 3-5 dias por semana, em todo o período de estudo. O idoso registrava num diário a atividade realizada (contagem de passos e duração da caminhada) medida por pedômetros. Já o programa do outro braço de estudo consistia em exercícios de treino de equilíbrio, fortalecimento muscular dos membros inferiores (baseados no programa de Otago) e Tai Chi; também este grupo foi instruído à prática destes exercícios no domicílio 3-5 dias por semana, e ao seu registro em diário.

O número de quedas e tropeços era ainda registrado. Os resultados foram apresentados por período de tempo e por grau de exposição (número de dias fisicamente ativos e contagem de passos): o grupo de marcha demonstrou uma redução no risco de queda, medida em quedas/pessoa ativa/dia $(R T I=0,38$, IC 95\% 0,19-0,77) e em quedas/passos/por pessoa ( $R T I=0,47$, IC 95\% 0,26-0,85) em relação ao grupo de treino de equilíbrio.

Contrariamente, o número de eventos em que se verificou tropeçar por pessoas/ano aumentou significativamente na caminhada ( $R T I=1,66$, IC 95\% 1,34-2,05). Estes resultados sugerem que treinos de marcha podem ser mais efetivos na prevenção de quedas, quando comparados com os treinos de equilíbrio, no indivíduo da comunidade, mas que não deverá ser recomendado aos indivíduos frágeis ou com elevado risco de queda, uma vez que induz mais episódios de tropeço. 


\section{Estudo coorte}

Um estudo de coorte ${ }^{14}$ prospectivo investigou o impacto da participação em classes preventivas de quedas "Steady as You Go (SAYGO)", na incidência de quedas. Esta classe consiste numa modificação do programa de exercício no domicílio Otago, liderada por um par, que foi identificado e eleito pelos colegas, após dez semanas iniciais ministradas por um instrutor.

Este estudo utiliza uma amostra de indivíduos da comunidade com idade igual ou superior a 65 anos, enviesada para o sexo feminino (189 mulheres vs. 18 homens), que participam nas classes semanais, com duração de 45 minutos. Os resultados obtidos demonstram uma taxa bruta de incidência de 0,75 quedas/pessoa/ano, sendo a incidência aos 12 meses inferior nos indivíduos que frequentavam o programa há 3 ou mais anos, em comparação os indivíduos com participações mais curtas (1 a 2 anos), (RTI=0,90, IC 95\% 0,82-0,99; p=0,03).

Da população de estudo, cinco idosos experienciaram mais de cinco quedas no período de seguimento, sendo que o resultado da taxa de incidência, após exclusão destes indivíduos, foi de 0,62 quedas/ pessoas/ano. Aos 12 meses, a participação na classe de exercício teve uma adesão de 69\%. Assim, o principal achado deste estudo foi a verificação de uma menor taxa de incidência de quedas nos indivíduos com participações nas classes SAYGO, por um prazo mais longo.

Meta-análise

Em 2016, Sherrington et al. ${ }^{15}$ atualizaram uma meta-análise prévia de avaliação do exercício na prevenção de quedas no idoso, contemplando estudos mais recentes. Os resultados reunidos indicaram um efeito do exercício na incidência de quedas no idoso da comunidade, expresso por RTI de 0,79 (IC 95\% 0,73-0,85; $p<0,001$; $\mathrm{I}^{2}=47 \%$ ). Este efeito manteve-se sobreponível em todas as análises de sensibilidade, mas a heterogeneidade diminuiu para $30 \%$, quando apenas estudos com baixo risco de viés (pontuação PEDro $\geq 7$ ) foram incluídos.

Efeitos mais significativos foram verificados nos estudos que incluíam intervenções com exercícios de treino de equilíbrio (RTI obtido pela meta-regressão de 0,85 (IC 95\% 0,73-0,995; p=0,04) e para uma duração semanal igual ou superior a 3 horas durante o período do programa implementado $(R T I=0,77, I C$ $95 \%$ 0,65-0,91, p=0,003, $\left.\left.\right|^{2} 61 \%\right)$.

Setenta e seis por cento da heterogeneidade foi explicada pela combinação destas duas variáveis, numa análise por meta-regressão. O efeito modelado nas quedas de programas de exercício que não contemplam o treino de equilíbrio e uma duração semanal superior a 3 horas foi de 0,90 (IC 95\% 0,82-0,99; $p=0,53$ ) e na presença de ambas, de 0,61 (IC 95\% 0,53-0,72; p<0,001), portanto, estimando uma redução da incidência de quedas em $39 \%$.

Em suma, os autores concluíram que o exercício como intervenção isolada pode prevenir quedas no idoso da comunidade, sendo que exercícios de treino de equilíbrio e maiores períodos de duração terão efeitos superiores, pelo que emitem as seguintes recomendações para esta população: os programas de exercício devem ter como objetivo treinos de equilíbrio de elevado grau de dificuldade, serem praticados por um período mínimo de 3h semanais; que a continuidade na participação destes programas é necessária para que os benefícios se mantenham; o exercício pode ser realizado em grupo ou de forma individual no domicílio, exercícios de fortalecimento e marcha podem ser adicionados, mas esta última não está recomendada no idoso com elevado risco de queda. 


\section{Discussão}

A elevada prevalência de utentes idosos nas listas de família, e o reconhecimento das quedas como importante causa de morbimortalidade, motivou as autoras à realização desta revisão, sobre a intervenção do exercício físico na prevenção de quedas neste grupo populacional.

Encontrando-se uma grande heterogeneidade clínica entre os indivíduos idosos, este trabalho foi dirigido ao utente sedentário, sem fragilidade, sem morbilidade grave, no contexto dos cuidados de saúde primários, isto é, em ambulatório, por caracterizar a maioria dos utentes deste grupo etário, nas listas de família.

Ao aplicarem-se rigorosamente os critérios de exclusão na seleção de estudos, é escassa a literatura publicada nos últimos 5 anos, exclusiva a esta população, o que justifica o número de estudos incluídos nesta revisão.

Quanto à análise dos resultados obtidos, todos os trabalhos selecionados mediam como resultado primário a incidência de quedas, o que traduz, logo à partida, um resultado orientado para o paciente, segundo a classificação SORT.

No que concerne à avaliação dos ECACs, o estudo ProAct65+ apresenta como limitações uma amostra inferior a $10 \%$ da população elegível e uma perda de seguimento de $53 \%$ aos 2 anos de follow-up, ainda que semelhante nos vários braços, pelo que a generalização dos resultados deve ser cuidadosa.

Todavia, os participantes voluntariamente recrutados apresentavam à partida uma predisposição à prática de exercício físico, pelo que serão com probabilidade representativos de uma população que frequentaria tais programas de exercício, se disponíveis. Ainda que indivíduos mais saudáveis e com melhor condicionamento físico fossem mais facilmente atraídos, note-se que cerca de um quarto dos participantes tinha história prévia de uma ou duas quedas.

Outra consideração deve ser tida em conta na interpretação dos resultados, uma vez que indivíduos com episódios de queda teriam menor probabilidade de entregar os seus diários, mas também esta percentagem de devoluções foi semelhante nos vários grupos de intervenção, pelo que o benefício demonstrado no grupo FaME não é atribuível a uma baixa notificação.

No braço OEP, não houve uma progressão suficiente da intensidade dos exercícios, o que pode explicar os resultados não significativos. É um ensaio metodologicamente bem desenhado, com análise de resultados por intenção de tratar, e um longo follow-up, atribuindo-se um nível de evidência 2.

O ECAC de Okubo et al. ${ }^{13}$ apresenta como limitações uma amostra pequena, a ausência de ocultação, e de uma análise por intenção de tratar, o que pode sobrestimar os resultados; ainda, um efeito preventivo significativo não seria encontrado se a análise não fosse realizada segundo a exposição. Estas variáveis - dias fisicamente ativos e número de passos - não refletem a evolução ao longo do período de seguimento, por terem sido medidas no momento pós-intervenção. Segundo a classificação SORT, este estudo é classificado com um nível de evidência 2.

Como pontos fortes, o estudo de coorte "SAYGO" mede uma intervenção "no mundo real", em contraste com a grande maioria dos ensaios clínicos randomizados. A taxa de incidência de quedas obtida é elevada, mas este resultado parece estar enviesado pelos cinco idosos que apresentaram quedas recorrentes, pois uma vez não contemplados, verifica-se uma diminuição da taxa de incidência para 0,62. 
Os autores defendem também que estes valores elevados são esperados, uma vez que a população de estudo apresenta elevado risco de queda, pela idade, antecedentes de queda, multimorbilidade e polifarmácia. Quanto à tendência demonstrada na redução de quedas com prática de exercício mais persistente, note-se que, após ajuste de variáveis confundidoras como sexo, idade e número de fármacos, indivíduos que frequentaram as classes por um período igual ou superior a 3 anos, apresentavam a 10\% menos probabilidade de queda.

Constituem ainda limitações metodológicas: o resultado primário de número de quedas ser recolhido por autorreporte, potenciando erros e viés de memória, a ausência de um grupo de controle, o reduzido tamanho amostral, com baixo poder para avaliação de quedas como outcome primário, e a generalização, por enviesamento da amostra para o sexo feminino; ainda o regime de frequência semanal de 45 minutos parece ser escasso, e um regime de frequência bissemanal poderia ter resultado numa incidência inferior. Pelas razões enumeradas, é atribuído um nível de evidência 2.

A revisão sistemática com meta-análise fornece uma forte evidência do exercício como intervenção única, na prevenção de quedas do idoso da comunidade. Os autores identificam como limitações a estimativa do efeito na meta-regressão, ter como base comparações não randomizadas entre estudos e uma interpretação potencialmente errada do tipo de exercício físico descrito nos estudos, para subsequente codificação.

Contudo, a confiança nos resultados obtidos é suportada pela estabilidade do efeito, quando metaanálise com modelo de efeito-fixo e testes de sensibilidade são realizados. Apesar da interpretação do gráfico em funil e teste de Egger sugerir a presença de viés, por influência de estudos de pequenas amostras, esses dados não são suportados pelos resultados da análise de sensibilidade na exclusão dos mesmos. Contemplando esta revisão estudos de 62 países diferentes, ainda que países subdesenvolvidos tenham menor representação, os resultados obtidos são suscetíveis de extrapolação para a generalidade desta população. Dada a consistência de resultados, a esta revisão sistemática atribuiu-se um nível de evidência 1.

Numa perspetiva global, os resultados deste trabalho demonstram o exercício físico como intervenção isolada efetiva na prevenção de quedas, que parece ser superior nos exercícios de treino de equilíbrio, realizados em grupo, com maior intensidade e por períodos mais longos. Ainda que o estudo de Okubo et al. ${ }^{13}$ demonstre uma superioridade da marcha relativamente aos treinos de equilíbrio, é o estudo com maiores limitações metodológicas, pelo que os seus resultados devem ser apreciados com alguma crítica.

Examinando as conclusões obtidas, especialmente segundo uma vertente da aplicabilidade na prática clínica, é essencial que as modalidades e regimes de exercício sejam adequados à realidade local.

O Tai Chi corresponde à modalidade mais frequentemente estudada no treino de equilíbrio, contudo, num ponto de vista cultural, não estará tão facilmente acessível em Portugal como nos países asiáticos ou até mesmo no Brasil. A marcha é um exercício sem custos, facilmente acessível à população, e culturalmente muito enraizada, pelo que se torna mais exequível.

Permanecendo alguma controvérsia relativa ao aumento de quedas com a prática deste exercício, determinada pela exposição ambiental, parece consensual que este aumento é verificado em indivíduos com elevado risco de queda, para os quais se encontra desaconselhada, mas que pode ser prescrita de forma criteriosa aos restantes indivíduos, de preferência em associação aos treinos de equilíbrio. 
A implementação de programas de exercício na comunidade acarreta custos, que serão indubitavelmente mais elevados se cumpridas as 3 horas semanais, como recomendado na meta-análise acima descrita, pelo que a prática e reprodução de exercícios no domicílio deverá ser fomentada, no sentido de alcançar a maior dose de exercício possível. Também a adesão a longo prazo pode ficar comprometida, sendo necessário encontrar um equilíbrio entre regimes de frequência e promoção de adesão. O estudo segundo o modelo SAYGO demonstrou que intervenções entre pares, semanais, podem otimizar adesão e dedicação a longo prazo.

\section{Conclusão}

O exercício físico é efetivo na prevenção de quedas no idoso da comunidade como intervenção única. Programas de exercício que contemplem treinos de equilíbrio e práticas semanais com duração igual ou superior a 3 horas apresentam melhores resultados (SORT A).

A manutenção de exercício físico moderado a intenso deve ser incentivado na prevenção de quedas a longo prazo (SORT B).

\section{Referências}

1. Serviço de Estudos sobre a População do Departamento de Estatísticas Censitárias e da População. O envelhecimento em Portugal Situação demográfica e sócio-econômica recente das pessoas idosas. Lisboa: Instituto Nacional de Estatística; 2002

2. Instituto Nacional de Estatística. Censos 2011 Resultados Definitivos - Portugal. Lisboa: Instituto Nacional de Estatística; 2012

3. World Health Organization. WHO Global Report on Falls Prevention in Older Age. [Internet]. Geneva: WHO; 2007 [acesso 07 Out 2017]. Disponível em: http://www.who.int/ageing/publications/Falls_prevention7March.pdf

4. Brasil. Ministério da Saúde. Estatuto do idoso. Brasília: Ministério da Saúde; 2009.

5. Rubenstein LZ. Falls in older people: epidemiology, risk factors and strategies for prevention. Age Ageing. 2006;35-S2:ii37-ii41. DOI: http://dx.doi.org/10.1093/ageing/afl084

6. Guideline for the prevention of falls in older persons. American Geriatrics Society, British Geriatrics Society, and American Academy of Orthopaedic Surgeons Panel on Falls Prevention. J Am Geriatr Soc. 2001;49(5):664-72.

7. Panel on Prevention of Falls in Older Persons, American Geriatrics Society and British Geriatrics Society. Summary of the Updated American Geriatrics Society/British Geriatrics Society clinical practice guideline for prevention of falls in older persons. J Am Geriatr Soc. 2011;59(1):148-57. DOI: http://dx.doi.org/10.1111/j.1532-5415.2010.03234.x

8. Inouye SK, Studenski S, Tinetti ME, Kuchel GA. Geriatric syndromes: clinical, research and policy implications of a core geriatric concept. J Am Geriatr Soc. 2007;55(5):780-91. DOI: http://dx.doi.org/10.1111/j.1532-5415.2007.01156.x

9. Falsarella GR, Gasparotto LPR, Coimbra AMV. Quedas: conceitos, frequências e aplicações à assistência ao idoso. Revisão da literatura. Rev Bras Geriatr Gerontol. 2014;17(4):897-910. DOI: http://dx.doi.org/10.1590/1809-9823.2014.13064

10. Sherrington C, Tiedemann A, Fairhall N, Close JC, Lord SR. Exercise to prevent falls in older adults: an updated meta-analysis and best practice recommendations. N S W Public Health Bull. 2011;22(3-4):78-83.

11. Gillespie LD, Robertson MC, Gillespie WJ, Sherrington C, Gates S, Clemson LM, et al. Interventions for preventing falls in older people living in the community. Cochrane Database Syst Rev. 2012;(9):CD007146.

12. Gawler S, Skelton DA, Dinan-Young S, Masud T, Morris RW, Griffin M, et al. Reducing falls among older people in general practice: The ProAct65+ exercise intervention trial. Arch Gerontol Geriatr. 2016;67:46-54. 
13. OkuboY, Osuka Y, Jung S, Rafael F, Tsujimoto T, Aiba T, et al. Walking can be more effective than balance training in fall prevention among community-dwelling older adults. Geriatr Gerontol Int. 2016;16(1):118-25. DOI: 10.1111/ggi.12444

14. Wurzer B, Waters DL, Hale LA, Leon de la Barra S. Long-term participation in peer-led fall prevention classes predicts lower fall incidence. Arch Phys Med Rehabil. 2014;95(6):1060-6. DOI: 10.1016/j.apmr.2014.01.018

15. Sherrington C, Michaleff ZA, Fairhall N, Paul SS, Tiedemann A, Whitney J, et al. Exercise to prevent falls in older adults: an updated systematic review and meta-analysis. Br J Sports Med. 2017;51(24):1750-8. DOI: 10.1136/bjsports-2016-096547

USF Santiago, ACeS Pinhal Litoral. Portugal. jobento13@ @otmail.com (Autora correspondente); nadinasousa @gmail.com 